\title{
Beam Spot Size Measurement Using Beamstrahlung Signals at the SLC Interaction Point
}

\author{
E. Gero \\ University of Michigan, Ann Arbor, Michigan 48109 \\ W. Kozanecki, P. Chen \\ Stanford Linear Accelerator Center, Stanford University, Stanford, California 94309
}

\section{Abstract}

We present a novel technique to measure separately the transverse sizes of $e^{+}$and $e^{-}$beams in linear colliders, that is based on analyzing patterns of beamstrahlung energy recorded while the two beams are scanned across each other. After establishing the principle of the method, we compare our predicted radiated energy profiles to actual measurements, and verify the consistency of our results with an independent beam size determination based on coherent mutual electromagnetic deflection of the two beams.

\section{Theory of Classical Beamstrahlung}

Consider a micron-sized electron bunch, containing about $10^{10}$ particles, colliding with a positron bunch at the interaction point (IP) of the Stanford Linear Collider (SLC). The strong coherent electromagnetic field generated by the positron (resp. electron) bunch as a whole, slightly inflects the trajectory of each incoming electron (resp. positron), and causes the latter to emit synchrotron radiation, called "beamstrahlung", as already observed experimentally [1].

Field strengths at the SLC IP are sufficiently low that classical beamstrahiung theory [2] fully describes the properties of the emitted radiation. These depend on the intensities $\left(N_{1}, N_{2}\right)$ of the two beams, their r.m.s. bunch lengths $\left(\sigma_{1 z}, \sigma_{2 z}\right)$, their energy $\left(E_{b}\right)$, their r.m.s transverse sizes $\left(\sigma_{1 x}, \sigma_{1 y}, \sigma_{2 x}, \sigma_{2 y}\right)$, and finally on the beam orientation parameters $(a, \alpha, \beta)$ (subscript 1 indicates the beam whose emitted radiation is being calculated, while subscript 2 refers to the beam producing the electromagnetic field responsible for the synchrotron emission). The charge population within each bunch is assumed to be gaussian in all three dimensions. For each of the two bunches separately, we choose a coordinate system such that the $z$ axis lies along the incoming beam direction, the $\mathrm{x}$ direction matches the major axis of the transverse beam profile (assumed elliptical), and the y-axis is aligned with its minor axis (the directions of the major and minor axis of the two beams need not coincide). $a$ is the radial distance between the centers of the two beams, $\alpha$ is the angle between the major axis of bunch 2 and the line connecting the bunch centers, and $\beta$ is the angle between the directions of the major axes of the two bunches.

The total number of photons radiated is given by [2]

$$
\begin{aligned}
& N_{1 \gamma}=\frac{5}{\sqrt{6 \pi}}\left(\frac{N_{1} N_{2} \alpha_{e} r_{e}}{\sigma_{1 x}}\right) B_{x} R_{1} R_{2} \\
& \quad \times \int_{-\infty}^{\infty} \int_{-\infty}^{\infty} f\left(\mu, \nu, B_{x}, R_{2}\right) e^{-g\left(\mu, \nu, \alpha, \beta, \xi, R_{1}\right)} d \mu d \nu
\end{aligned}
$$

where

$$
\begin{array}{r}
f\left(\mu, \nu, B_{x}, R_{2}\right)=\frac{1}{\sqrt{R_{2}^{2}-1}} \mid w\left(\frac{B_{x} R_{2}(\mu+i \nu)}{\sqrt{R_{2}^{2}-1}}\right) \\
-e^{-B_{x}^{2}\left(\mu^{2}+R_{2}^{2} \nu^{2}\right)} w\left(\frac{B_{x}\left(\mu+i R_{2}^{2} \nu\right)}{\sqrt{R_{2}^{2}-1}}\right) \mid \\
g\left(\mu, \nu, \alpha, \beta, \xi, R_{1}\right)=(\mu \cos \beta+\nu \sin \beta-\xi \cos (\beta-\alpha))^{2} \\
+R_{1}^{2}(-\mu \sin \beta+\nu \cos \beta+\xi \sin (\beta-\alpha))^{2}
\end{array}
$$

Here, $\alpha_{e}$ is the fine structure constant and $r_{e}$ is the classical radius of the electron; $R_{1}$ and $R_{2}$ are the aspect ratios of the bunches $\left(R_{1}=\sigma_{1 x} / \sigma_{1 y}, R_{2}=\sigma_{2 x} / \sigma_{2 y}\right)$; and $B_{x}$ and $B_{y}$ are the beam size ratios $\left(B_{x}=\sigma_{1 x} / \sigma_{2 x}, B_{y}=\right.$ $\left.\sigma_{1 y} / \sigma_{2 y}\right) . \quad w(z)$ is the complex error function: $w(z)=$ $\exp \left(-z^{2}\right) \operatorname{erfc}(-i z) . \mu$ and $\nu$ equal $x / \sqrt{2} \sigma_{1 x}$ and $y / \sqrt{2} \sigma_{1 x}$, respectively, and $\xi$ equals $a / \sqrt{2} \sigma_{1 x}$.

The total energy emitted can be expressed as

$$
\begin{aligned}
U_{1} & =\frac{4}{3 \sqrt{\pi}}\left(\frac{N_{1} N_{2}^{2} r_{e}^{3} \gamma^{2} m c^{2}}{\sigma_{1 x} \sigma_{1 y} \sigma_{2 z}}\right) B_{x}^{2} R_{2}^{2} \\
& \times \int_{-\infty}^{\infty} \int_{-\infty}^{\infty} f^{2}\left(\mu, \nu, B_{x}, R_{2}\right) e^{-g\left(\mu, \nu, \alpha, \beta, \xi, R_{1}\right)} d \mu d \nu
\end{aligned}
$$

where $\gamma$ is the relativistic factor, $m$ is the mass of the electron, and $c$ is the speed of light.

The maximum critical energy is

$$
\left(E_{c}\right)_{\max }=3 f_{7 n}\left(B_{x}, R_{2}\right)\left(\frac{N_{2} r_{e}^{2} \gamma^{2} m c^{2}}{\alpha_{e} \sigma_{1 x} \sigma_{2 x}}\right) B_{x} R_{2}
$$

where $f_{m}\left(B_{x}, R_{2}\right)$ is the maximum magnitude of the electric field of bunch 2 in units of $\left(e N_{2} / \sigma_{2 x} \sigma_{2 z}\right)$. 
Finally, the photon energy spectrum can be calculated :

$$
\frac{d N_{1 \gamma}}{d E_{\gamma}}=\frac{\sqrt{6}}{3 \pi^{2}}\left(\frac{N_{1} \alpha^{2} \sigma_{2 z}}{r_{e} \gamma^{2} m c^{2}}\right) R_{1} I\left(R_{1}, R_{2}, B_{x}, \alpha, \beta, \xi, \epsilon\right)
$$

where

$$
\begin{array}{r}
I\left(R_{1}, R_{2}, B_{x}, \alpha, \beta, \xi, \epsilon\right)=\int_{-\infty}^{\infty} \int_{-\infty}^{\infty} \int_{\epsilon h\left(\mu, \nu, B_{x}, R_{2}\right)}^{\infty} e^{-g\left(\mu, \nu, \alpha, \beta, \xi, R_{1}\right)} \\
\times K_{5 / 3}(s) \sqrt{\ln \left(\frac{s}{\epsilon h\left(\mu, \nu, B_{x}, R_{2}\right)}\right)} d s d \mu d \nu(7)
\end{array}
$$

and

$$
h\left(\mu, \nu, B_{x}, R_{2}\right)=\frac{f_{m}\left(B_{x}, R_{2}\right)}{f\left(\mu, \nu, B_{x}, R_{2}\right)} \quad \epsilon=\frac{E_{\gamma}}{\left(E_{c}\right)_{\max }}
$$

\section{Beamstrahlung Radiation DETECtor}

Beamstrahlung photons emitted by electrons and positrons impinge on two detectors, located symmetrically on either side of the SLC IP, downstream of the first bending dipole encountered by the outgoing charged particle bearns. In this section, we recall the principle and construction of these "beamstrahlung monitors", outlining as we proceed the physical processes that convert the beamstrahlung energy fiux into a measurable electrical signal.

Each monitor $[2,3]$ consists of a Čerenkov counter filled with ethylene gas at a pressure of 0.3 atmospheres. Beamstrahlung photons incident on the monitor first hit a converter plate, where about $3 \%$ of them (for $E_{\gamma}=50 \mathrm{MeV}$ ) convert into $e^{+} e^{-}$pairs. The Čerenkov light produced in the gas by the conversion products is then reflected by a field mirror onto a plane mirror, that guides it into a light channel, consisting in turn of a periscope with two $90^{\circ}$ reflections. This light channel ends in five side-byside vacuum windows of fused silica, held by a stainless stee] jacket. Downstream of the windows, the light hits five Hamamatsu R580 photomultiplier tubes, whose output signals are integrated, digitized, and summed together.

The total charge collected from the phototubes, expressed in terms of ADC counts, is related to the total number of beamstrahlung photons radiated, by $[2,4]$

$$
N_{A D C}=C \int_{E_{\text {cof }},}^{E_{c+n}} \frac{d N_{1 \gamma}}{d E_{\gamma}} N_{c}\left(E_{\gamma}\right) f_{\gamma} d E_{\gamma}
$$

where $C\left(\approx 4.5 \times 10^{-5}\right)$ is the number of $\mathrm{ADC}$ counts per Čerenkov photon detected, $d N_{1 \gamma} / d E_{\gamma}$ is the number of beamstrahlung photons produced at energy $E_{\gamma}$, and $N_{c}\left(E_{\gamma}\right)$ is the average number of Cerenkov photons produced by a converted beamstrahlung photon of energy $E_{\gamma}$ :

$$
\begin{aligned}
N_{c}\left(E_{\gamma}\right)=\frac{4 \pi \alpha l \Delta \nu}{c E_{\gamma}} & {\left[\left(1-\frac{1}{n^{2}}\right)\left(E_{\gamma}-E_{c o f f}\right)\right.} \\
& \left.+\frac{m^{2} c^{4}}{n^{2}}\left(\frac{1}{E_{\gamma}}-\frac{1}{E_{\text {coff }}}\right)\right]
\end{aligned}
$$

Here $l$ is the path length $(21 \mathrm{~cm})$ of a conversion electron or positron in the counter gas, $n$ is the index of refraction of this gas (1.0002), $\Delta \nu$ is the frequency interval over which the phototubes are sensitive $\left(8.57 \times 10^{14} \mathrm{~Hz}\right)$, and $E_{\text {coff } f}$ is the Cerenkov threshold energy (approximately $26 \mathrm{MeV}$ ),

$$
E_{\text {coff }}=\frac{n m c^{2}}{\sqrt{n^{2}-1}}
$$

Finally, $f_{\gamma}\left(E_{\gamma}\right)$, the fraction of photons that pair produce, is given by

$$
f_{\gamma}\left(E_{\gamma}\right)=\left(1-e^{-N \sigma\left(E_{\gamma}\right) x}\right)
$$

where $N$ is the number of nuclei per unit volume, $x$ is the thickness of the converter plate $(0.79 \mathrm{~mm})$, and $\sigma\left(E_{\gamma}\right)$ is the pair production total cross section per nucleus at energy $E_{\gamma}$, calculated using the Racah formula for pair production in the field of an unscreened point nucleus [2].

\section{SPOT SIZE MEASUREMENT TECHNIQUE}

We can now predict and measure the total energy (convoluted with the acceptance, threshold and resolution functions of the beamstrahlung monitor) radiated by either beam. Our spot size measurement technique $[2,5]$ fundamentally relies on the fact that the energy pattern radiated by one of the bunches depends, in different ways, both on its own charge distribution (i.e. on the spatial distribution of the radiating particles), and on that of the other beam (which determines the distribution of electromagnetic fields that induce the radiation process). By measuring the radiation patterns from both beams simultaneously as these are scanned across each other (thereby probing each other's charge distribution and field configuration), it is possible to unfold, in a global fit to these patterns, the parameters describing the transverse bunch shapes. As no attempt is made to measure the absolute magnitude of the beamstrahlung flux, variables such as bunch length or beam current, which greatly affect the energy and intensity of the photon fiux, but not its spatial pattern, become largely irrelevant (except through their coupling with Cerenkov threshold effects).

In practice, the method consists in holding one of the beams fixed, and scanning the other beam transversely across it, first horizontally, and then vertically, using steering dipoles located on either side of the SLC IP. Each scan extends well beyond several beam radii, in order to probe the full spatial extent of the electromagnetic field of the bunch, and typically covers a range of $\pm 40 \mu \mathrm{m}$, in $2 \mu \mathrm{m}$ steps $[1,2]$. At each scan point, the beamstrahlung flux (eq. 9) is recorded and averaged over 10 consecutive beam pulses; the beam is then moved to the next scan point, and the whole procedure is repeated. Such a "beamstrahlung scan" therefore results in a total of four beamstrahlung patterns, which can be adequately modelled by our beamstrahlung and detector simulation, as illustrated in fig. 1. 

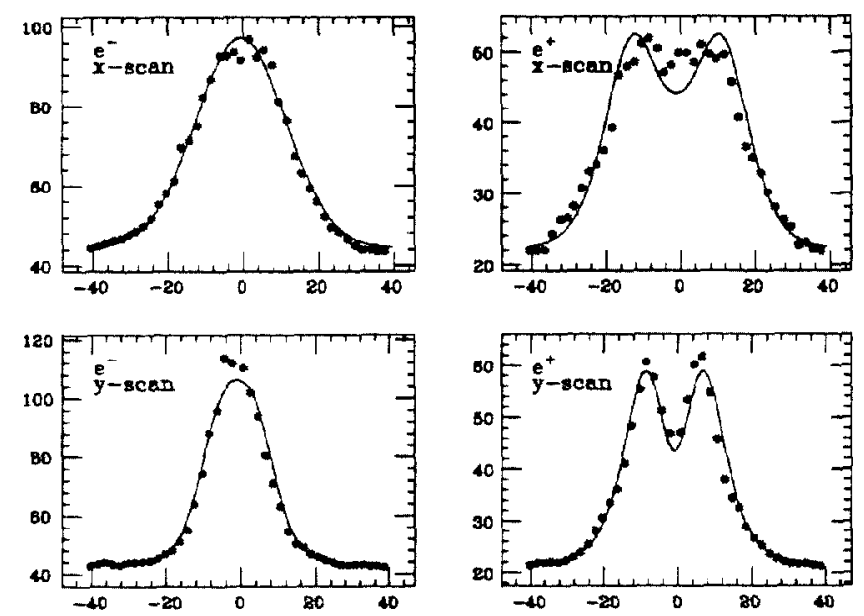

Figure 1: Number of detected Cerenkov photons (ADC counts) from radiating electrons (left) and positrons (right), vs horizontal (top) or vertical (bottom) distance between the colliding bunches, for the data (points) and the simulation (curves).

One of the simplest shape parameters that characterizes such a beamstrahlung pattern is its $2-\sigma$ truncated r.m.s width $W[2]$. We can extract from the 4 radiation patterns above, 4 measured width parameters $W_{1 x}^{m}, W_{1 y}^{m}, W_{2 x}^{m}$ and $W_{2 y}^{m}$. If both beams are upright, i.e. if their major and minor axes are all either horizontal or vertical, then 4 numbers suffice to describe the transverse profiles of the two beams, viz. the r.m.s beam sizes $\sigma_{1 x}, \sigma_{1 y}, \sigma_{2 x}$, and $\sigma_{2 y}$. It should therefore be possible to relate these 4 transverse beam sizes $\sigma$ to the 4 width parameters $W$, and to thereby measure the beam sizes by matching the expected values of the width parameters $W$ to their observed values.

To this effect, we first construct a "library" of beamstrahlung scans, by generating, according to the prescriptions of section II, several sets of simulated beamstrahlung patterns like the ones of fig. 1, that cover a sufficiently wide range of possible beam size combinations. For each of the generated combinations, the widths parameters are evaluated from the simulated beamstrahlung patterns and stored in a four-dimensional look-up table. This allows, given any combination of assumed values for $\sigma_{1 x}, \sigma_{1 y}, \sigma_{2 x}$, and $\sigma_{2 y}$, to calculate by interpolation, the expected values of the width parameters $W_{1 x}, W_{1 y}, W_{2 x}$, and $W_{2 y}$.

The four beam sizes $\sigma_{1 x}, \sigma_{1 y}, \sigma_{2 x}$ and $\sigma_{2 y}$ are then "measured" by finding the set of values of $\sigma$ 's that minimize the $x^{2}$-like quantity

$$
\begin{aligned}
\chi^{2} & =\left(\frac{W_{1 x}^{m}-W_{1 x}}{W_{1 x}^{m}}\right)^{2}+\left(\frac{W_{1 y}^{m}-W_{1 y}}{W_{1 y}^{m}}\right)^{2} \\
& +\left(\frac{W_{2 x}^{m}-W_{2 x}}{W_{2 x}^{m}}\right)^{2}+\left(\frac{W_{2 y}^{m}-W_{2 y}}{W_{2 y}^{m}}\right)^{2}
\end{aligned}
$$

where $W_{1 x}, \ldots, W_{2 y}$ are the expected values of the width parameters, corresponding to the assumed values of $\sigma_{1 x}, \ldots, \sigma_{2 y}$. Each $W$ is a function of all four $\sigma$ s.

The validity of the method has been tested by measuring, simultaneously with the beamstrahlung patterns, the electromagnetic deflection induced by one bearn on the other [6]. This technique directly nneasures the "interaction radii" $\Sigma_{x}=\sqrt{\sigma_{1 x}^{2}+\sigma_{2 x}^{2}}$ and $\Sigma_{y}=\sqrt{\sigma_{1 y}^{2}+\sigma_{2 y}^{2}}$, but is unable to separate the individual electron $\left(\sigma_{1}\right)$ and position $\left(\sigma_{2}\right)$ contributions to $\Sigma_{x}$ and $\Sigma_{y}$. We compare in fig. 2 the interaction radii as measured by the beambeam defiections and by the beamstrahlung patterns, in a controlled experiment where all bearn parameters are kept constant, except for the horizontal size of the electron beam which is varied using adjustabie quadrupole magnets. As the longitudinal position of the horizontal electron waist is varied around the nominal (zero) position, $\Sigma_{y}$ stays approximately constant (as it should), while $\Sigma_{x}$ varies by the expected amount. Beamstrahlung and deflection measures of the spot sizes yield results consistent with cach other, both qualitatively and quantitatively.


Figure 2: $\Sigma_{x}$ and $\Sigma_{y}$ vs. position of the horizontal $e^{-}$ waist, as measured by beam-beam deflections (*) and by beamstrahlung patterns $(+)$.

\section{REFERENCES}

[1] G. Bonvicini et. al., "First Observation of Beamstrahlung," Phys. Rev. Lett., vol. 62, pp. 2381-2384, May 1989.

[2] E. Gero, "The Theory and Observation of Classical Beamstrahlung at the SLC," University of Michigan, Ann Arbor, Michigan, Ph.D. dissertation, 1991.

[3] G. Bonvicini et. al., "A Monitor for Gamma Radiation at at Zero Degrees from the SLC Collision Point," Nucl. Instr, and Meth. A, vol. 277, pp. 297-303, May 1989.

[4] P. Chen, "Can One Measure Beam Size with Beamstrahlung Monitor at SLC?" SLAC-AAS-40, Nov. 1988.

[5] E. Gero et. al., "Beamstrahlung as an Optics Tuning Tool at the SLAC IP," in 1989 IEEE Particle Accelerator Conference, Chicago, Ill., Mar. 1989, pp. 1542-1543.

[6] W. Koska et. al., "Beam-beam Deflection as a Beam Tuning Tool at the SLAC Linear Collider." Nucl. Instr. and Meth. A, vol. 286, pp. 32-36, Jan. 1990. 